\title{
Calibration of Immunofluorescence: a standardization approach
}

\author{
Vishnu Prasad Ravitla ${ }^{1}$, Boris Radau ${ }^{2}$, Golam Abu Zakaria ${ }^{1,3}$ \\ ${ }^{1}$ Biomedical Engineering Master Studies of HS-Anhalt and Martin Luther University, Halle-Wittenberg, Department of \\ Electrical, Mechanical and Industrial Engineering, Anhalt University of Applied Sciences, Köthen, Germany, \\ vishnu.ravitla@student.uni-halle.de \\ ${ }^{2}$ Medipan GmbH, Dahlewitz/Berlin, Germany \\ ${ }^{3}$ Dept. of Medical Radiation Physics, Gummersbach Hospital, Academic Training Hospital of the University of Co- \\ logne, Gummersbach, Germany
}

\section{Structure: $\quad$ 1. Introduction / 2. Principle of Operation / 3. Results / 4. Conclusion}

\begin{abstract}
Objective of this topic is to develop a software module for the calibration of automatic cell based immunofluorescence microscopes. Developing software comes in two steps, primarily developing a new procedure for scanning slides and, secondarily image processing. The software is written in Microsoft Visual Studio by utilizing already existing drivers for hardware components Camera, LED, Microscope and Stage. Software code is written in C++ programming language. Additionally wxWidgets is used (cross-platform GUI and tools). The calibration software produces a set of measurements from a slide in terms of intensity levels present on the slide, by calculating the number of pixels present in the image. Along with the intensity values a list of hardware settings are displayed. Template matching technique is implemented for standardization of starting position of stage in various machines.
\end{abstract}

\section{Introduction}

Cell based Immunofluorescence microscopy is a technique used primarily on biological samples. To evaluate the samples in a routine environment a fully automated microscope system is required. The automated system consists of LED light source, motorized microscope, sample table (stage), camera and software. The software used is AKLIDES. The calibration software produces a set of measurements from a slide in terms of intensity levels present on the slide, by calculating the number of pixels present in the image. Along with the intensity values a list of hardware settings are displayed, that is useful to adjust various systems work precisely. It is based on precisely localizing the same starting position of scan for all AKLIDES systems and generating device parameters for each scan step.

\subsection{AKLIDES}

AKLIDES is a fully automated system for the evaluation of cell - based immunofluorescence tests. It is based on an automated fluorescence microscope equipped with a motorized sample table, LED light source, camera and software. AKLIDES features include fully automated cell based screening and evaluation of cell based indirect immunofluorescence tests, pattern specific quantification, live imaging, and archiving of results in internal database.

\subsection{Challenges with AKLIDES}

There are four primary factors affecting the results in terms of intensity from AKLIDES. Quality of the images and precision of the system is assessed by these factors. Taking influential factors into consideration the calibration software is designed in a way to produce precise

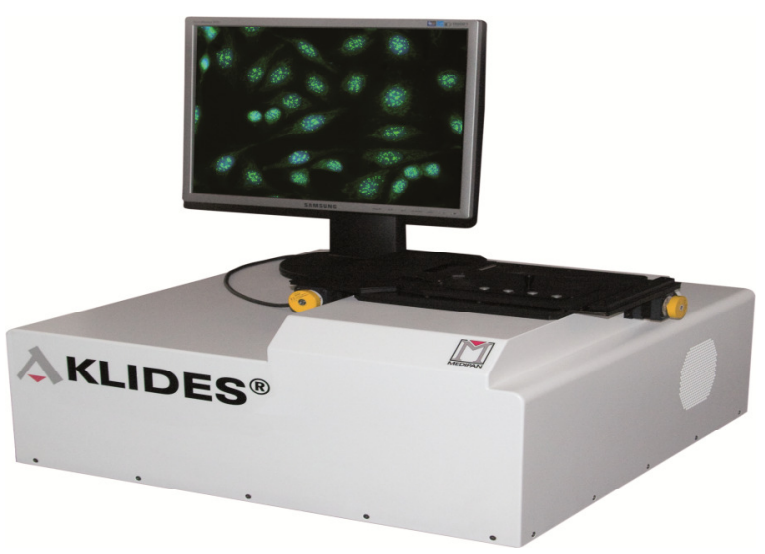

Image 1 AKLIDES (automated immunofluorescence system)

results. The software includes certain techniques that can correlate these factors. The factors that can influence the resulting images and their data are listed below.

- Camera exposure time

- LED Intensity (Power)

- Microscope objective focus (' $Z$ ' position)

- Stage startup point setup

\subsection{Calibration Software}

Calibration Software is developed in Microsoft Visual $\mathrm{C}++$ environment. The program code is in $\mathrm{C}++$, and HTML components are embedded into the $\mathrm{C}++$ code environment, it also uses wxWidgets to develop Calibration software GUI that uses native windows. This software is available in two versions with respect to the number of 
LED's in the fluorescence system, one version for use with two LED systems and another version for four LED systems.

There are two modes of operation, one is live image mode and the other one is scan mode. Live image mode is used to view the current image of the sample under the microscope along with the histogram of the image. Scan mode generates a set of data containing scanned images, intensity values (useful pixel values) in the scanned images, and optimum device settings for each step of scan.

\section{Principle of Operation}

The basic principle behind the calibration software is divided into two segments. At first the software connects and checks status of all the hardware devices (Camera, LED, Microscope, and Stage). Scanning of a slide using this software is started with selection of a profile (section 2.1). After starting scan, stage is moved to a starting point given in the profile, and sets microscope objective at a minimum magnification level and selects LED channel. Current image is displayed on the screen. Template matching technique is used at this stage (e.g. Image 2). The software loads templates from the database and locates the same pattern in the current image and calculates the position(X/Y coordinates). Stage will be moved till the difference between positions of the template in current image and reference image is equal to zero. This process is repeated for all the available magnification levels till the desired position reached.

At the second level after finishing the table setup procedure, an actual scan of the slide will be started, during this process the software tunes automatically (section 2.3) camera exposure time, microscope objective position (' $\mathrm{Z}$ ' position), and LED intensity (exposed to two LED channels e.g. Image 3 ) to get an optimum image. This image is captured, saved and pixel count (image intensity) of that image will be calculated. This process will be repeated for the number of images to be taken from a single slide. At the end detailed reports will be generated. The calibration software can generate reports of type PDF, HTML, and Text.
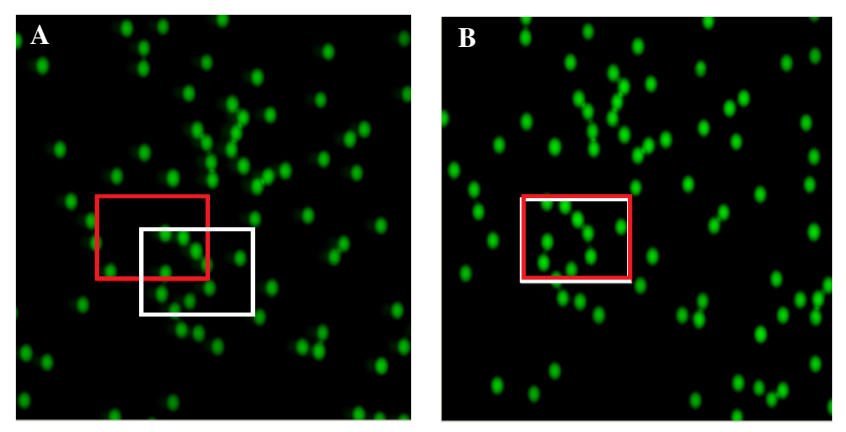

Image 2 Stage positioning: target area (fixed) is under red colored rectangle; template area (mobile) is under white colored rectangle. (A) Stage target area not reached (B) target area reached
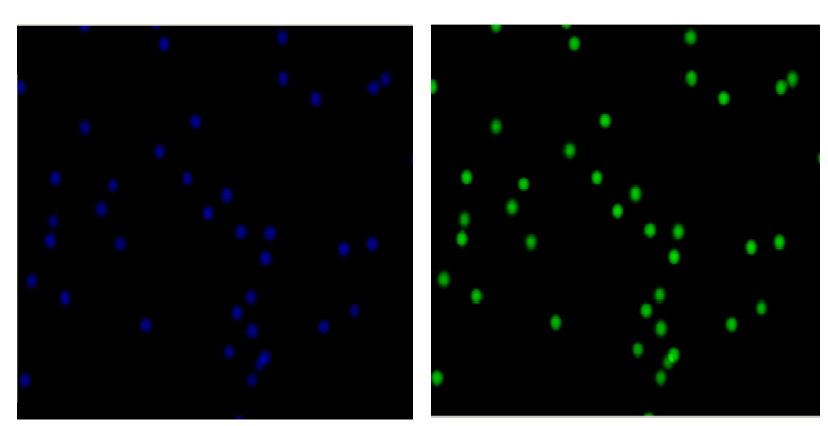

Image 3 Sample images of a scanned calibration slide with LED light source at $400 \mathrm{~nm}$ (blue) and $490 \mathrm{~nm}$ (green)

\subsection{Intensity Slide Profiles}

An intensity slide profile (profile) is a set of data containing images and settings to perform a scan of a specific slide. These profiles can be generated using the calibration software. A valid profile contains six image files and a settings ('.ini') file.

Template extraction technique is implemented to generate user defined profiles. A profile contains three reference images at each magnification level present in the microscope, and three templates each from respective reference images. An initialization file ('.ini'), optimum device settings (parameters) for optimum intensity levels in the slide, is also generated along with the profile. Each profile is named to its slide Id number. During scanning of a slide, profile is selected using its Id number and slide image type displayed in the profile dialog box.

\subsection{Template Matching}

Template matching technique uses a convolution mask (template), tailored to a specific feature of the search image, which has to be detected. This technique can be performed easily on grey scale images or edge images. The convolution output will be highest at places where the image structure matches the mask structure, where large image values get multiplied by large mask values.

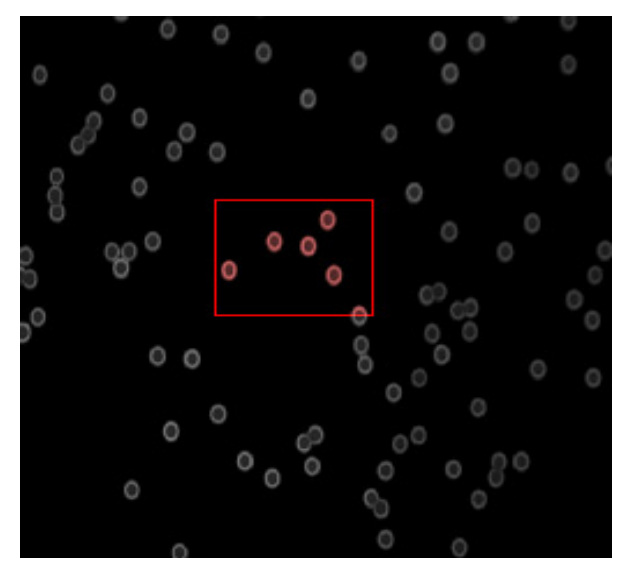

Image 4 Template matching (area under the rectangle is the mask structure matching) 


\subsection{Auto Tuning}

Each device present in the automated system has different variables (given as factors under section 1.2). All these variables have to be controlled relatively to get an optimum image with optimum intensity. Camera exposure time, LED intensity, and microscope objective focus varied with respect to histogram of the current image. Histogram of the current image is computed with total number of pixels, pixels at maximum brightness level (i.e. [255]), and pixels at minimum brightness level (i.e. [0]). All the above three parameters of camera, LED, and microscope are tuned in response to number of useful pixels in the histogram till an optimum image is displayed.

\section{Results}

Quality of the image is assessed by its intensity, i.e. number of useful pixels present on the image by subtracting noise and dark background.

An experiment with two different types of slides was conducted on the machines with AKLIDES software and Calibration software for calculating deviation.

Results from AKLIDES and Calibration Software are compared in the Table 1; deviation in the AKLIDES is more due to factors affecting intensity of the images described in section 1.2, whereas deviation with calibration software is significantly less resulting in precise measurement of slides under microscope.

\begin{tabular}{|c|c|c|c|}
\hline \multirow{2}{*}{\multicolumn{2}{|c|}{ Image Intensity Results }} & \multirow{2}{*}{\multicolumn{2}{|c|}{$\begin{array}{c}\begin{array}{c}\text { Relative Standard De- } \\
\text { viation }(\%)\end{array} \\
\text { Slide Type }\end{array}$}} \\
\hline & & & \\
\hline & & $\mathbf{A}$ & B \\
\hline \multirow{2}{*}{ AKLIDES } & $\begin{array}{l}\text { Within the } \\
\text { machine }\end{array}$ & 9.93 & 16.66 \\
\hline & $\begin{array}{l}\text { Among the } \\
\text { machines }\end{array}$ & 18.46 & 16.35 \\
\hline \multirow{2}{*}{$\begin{array}{l}\text { Calibration } \\
\text { Software }\end{array}$} & $\begin{array}{l}\text { Within the } \\
\text { machine }\end{array}$ & 0.33 & 1.42 \\
\hline & $\begin{array}{l}\text { Among the } \\
\text { machines }\end{array}$ & 4.46 & 5.33 \\
\hline
\end{tabular}

Table 1 Comparison of ' $\%$ ' deviation within one machine and among five machines using AKLIDES and Calibration software

\section{Conclusion}

With the completion of this project, AKLIDES software now has an important tool to optimize different automated fluorescence microscopes that uses discrete device parameters. A standardization procedure is now available for AKLIDES machines in different work environments on the basis of non bleaching fluorescent microparticles.

During the development process of the calibration software, the following three questions were answered:
1. What is the best practice for making the stage (microscope table) movements precisely enough to find the same starting position for each measurement?

2. Implement a procedure to tune the devices (Camera, LED, Microscope and Stage) settings automatically?

3. Create a report of optimized device parameters for each measurement?

\section{References}

[1] Isaac, Bankman: Handbook of Medical Image Processing and Analysis: Academic Press, 2008

[2] Soda, Rigon: Automatic Acquisition of Immunofluorescence Images: CMBS, 2006

[3] Petrou \& Gracia: Image Processing. Dealing with Textures: Wiley, 2006

[4] Robert, Price: Microscopy and Microanalysis: Cambridge University Press, 2009

[5] James, Allert: Programming with Visual C++: Course Technology, 2008

[6] Bjarne, Stroustrup: The C++ Programming Language: Addison-Wesley Longman, 1997

[7] Internal Resources:Software Drivers, Images: Medipan GmbH, 2011

[8] Microscopy Articles (2011): Microscopy objective focal length. [Online]. Available: http://www.prioruk.com/productinfo_auto_focus_focu smotors.html/

[9] Microscopy Analysis (2011): LED light sources. [Online]. Available: http://www.microscopyanalysis.com/features/

[10] Kappa Optronics (2011): Kappa camera. [Online]. Available: http://www.kappa.de/

[11] Märzhäuser (2011): Motorized microscope stages. [Online]. Available: http://www.marzhauser.com/ 